\title{
Spectral Properties and Interpolation Error Analysis for Variable Sample Rate Conversion Systems
}

\author{
Andre Tkacenko \\ Signal Processing Research Group \\ Jet Propulsion Laboratory, California Institute of Technology \\ 4800 Oak Grove Drive, Pasadena, CA 91109 \\ E-mail: Andre.Tkacenko@jpl.nasa.gov
}

\begin{abstract}
The problem of variable sample rate conversion (SRC) has received much attention on account of its applications in software defined radios (SDRs) that must support a wide variety of data rates. In this paper, we investigate the spectral properties of variable SRC and focus on the interpolation error obtained using any two interpolation kernels. We show that SRC is a generalization of decimation for both rational and irrational conversion ratios. In addition, a frequency domain expression for the mean-squared interpolation error is derived and simplified. Simulations presented show the degradation effects of using practical piecewise polynomial based interpolants as opposed to the underlying bandlimited sinc function for several input signals.
\end{abstract}

\section{INTRODUCTION}

A primary challenge facing the development of software defined radios (SDRs) is the ability to accommodate a variety of data rates subject to fixed system architectures [1], [2]. Examples of these architectures are the analog-to-digital converter (ADC) used at the front end and the tracking loops (carrier synchronization, symbol timing, etc.) used subsequently.

To conform to both of these types of fixed systems pragmatically, the sample rate must be converted digitally. This can be achieved using a variable sample rate conversion (SRC) system [2]. An intermediate frequency (IF) SDR receiver employing variable SRC is shown in Fig. 1. In this system, an analog datamodulated signal at data rate $R$ is sampled at a fixed rate $F_{s}$ to produce a real digital IF signal. Upon quadrature conversion, a complex baseband signal sampled at rate $F_{s}^{\prime}=\frac{F_{s}}{2}$ is formed. The signal of interest is then centered at zero frequency using a tunable numerically controlled oscillator (NCO).

At this point, the sample rate must be altered to fit the fixed redundancy factor (\# of samples/symbol) stipulated for the tracking loops. For example, if the loops require a redundancy of $K$ as in Fig. 1, the sample rate coming into them should be $F_{s}^{\prime \prime}=K R$. The purpose of the variable SRC system is to adjust this rate from $F_{s}^{\prime}$ to $F_{s}^{\prime \prime}$. Prior to this, the SRC system should also remove out-of-band artifacts to prevent aliasing.

In this paper, we first focus on the spectral properties of variable SRC for the interpolation kernel signal model typically assumed [2]. Though this model has received much attention in the literature (i.e., see [3], [4], [5]), most of the consideration has consisted of time domain analysis. With the derived

The research described in this publication was carried out at the Jet Propulsion Laboratory, California Institute of Technology, under a contract with the National Aeronautics and Space Administration.

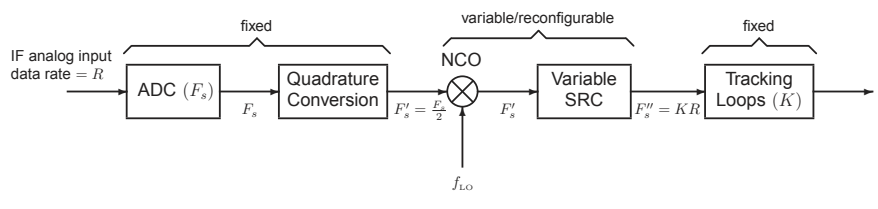

Fig. 1. Block diagram of an IF SDR receiver employing variable SRC.

spectral characterization of the variable SRC output, we show the relation between SRC and decimation [6]. Specifically, we show that variable SRC is a generalization of decimation for both rational and irrational rate conversion ratios.

We then turn our attention to interpolation error analysis for the variable SRC signal model. Using the spectral properties of this model, we derive a simplified frequency domain expression for the mean-squared interpolation error for any two interpolation kernels. Simulation results presented elucidate the degradation associated with using practical piecewise polynomial based interpolation kernels instead of the underlying bandlimited sinc function. In line with intuition, we show that as the output rate conversion factor increases, so that the input samples appear less redundant, the error increases. It is also shown that for a fixed rate conversion factor, higher order interpolation kernels always outperform lower order ones.

\section{A. Outline}

In Sec. II, we review the interpolation kernel signal model assumed throughout the paper. The variable SRC problem is explored in Sec. III. Spectral properties of the variable SRC output are derived in Sec. IV, where the connection between variable SRC and decimation is established. In Sec. $\mathrm{V}$, we derive a simplified frequency domain expression for the mean-squared interpolation error. Simulation results of the interpolation error are presented in Sec. VI for several practical scenarios. Concluding remarks are made in Sec. VII.

\section{B. Notations}

All notations used are as in [7]. In particular, continuoustime (analog) and discrete-time (digital) normalized frequencies are denoted as $F$ and $f$, respectively. Parentheses and square brackets are respectively used for continuous-time and discrete-time function arguments. For example, $x(t)$ would denote a continuous-time function for $t \in \mathbb{R}$, whereas $y[n]$ would denote a discrete-time function for $n \in \mathbb{Z}$. 


\section{Interpolation KeRnel Signal Model}

All equivalent complex baseband analog signals input to the system of Fig. 1 are assumed to be of the following form [2].

$$
x(t)=\sum_{k=-\infty}^{\infty} c_{d}[k] h\left(\frac{t}{T}-k\right)
$$

The signal model of (1) is referred to as the interpolation kernel signal model [2] where $h(t)$ denotes the dilated interpolation kernel [2], $T$ represents the kernel spacing interval, and $c_{d}[k]$ is the basis coefficients sequence [2].

Several interesting classes of signals are generated using the model given in (1) [8]. For example, from the Nyquist sampling theorem [6], if $x(t)$ is bandlimited to $F_{B L}$ (meaning $X(j 2 \pi F)=0$ for $\left.|F| \geq F_{B L}\right)$, then $x(t)$ is of the form,

$$
x(t)=\sum_{n=-\infty}^{\infty} x\left(n T_{s}\right) \operatorname{sinc}\left(\frac{t}{T_{s}}-n\right)
$$

where the sinc function is defined as $\operatorname{sinc}(x) \triangleq \frac{\sin (\pi x)}{\pi x}$ and $T_{s}$ is given by $T_{s}=\frac{1}{2 F_{B L}}$. Comparing (2) to (1), it can be seen that all bandlimited signals can be expressed in terms of the interpolation kernel model where we have,

$$
h(t)=\operatorname{sinc}(t), c_{d}[k]=x\left(k T_{s}\right), T=T_{s}
$$

For this reason, any signal bandlimited to $F_{B L}$ can be recovered from uniform sampling at a rate of $F_{s}=\frac{1}{T_{s}}=2 F_{B L}$ (called the Nyquist rate [7], [6]), as this will automatically yield the basis coefficients sequence $c_{d}[k]$ as seen from (3).

After uniform sampling of the signal $x(t)$ from (1), which is attained here after quadrature conversion of the ADC output, we will have access to the sequence $x_{d}[n] \triangleq x\left(n T_{s}\right)$, where $T_{s}$ denotes the sampling interval. For the remainder of this paper, we will assume that $T_{s}=T$. Then, from (1), we have,

$$
x_{d}[n]=x\left(n T_{s}\right)=\sum_{k=-\infty}^{\infty} c_{d}[k] h(n-k)=c_{d}[n] * h_{d}[n]
$$

where the discrete-time sequence $h_{d}[n]$ is defined as $h_{d}[n] \triangleq$ $h(n)$. In other words, from (4), $x_{d}[n]$ is simply the discretetime convolution of the basis coefficients sequence $c_{d}[n]$ with the sampled interpolation kernel sequence $h_{d}[n]$.

To reconstruct the original analog signal $x(t)$ from (1) at some prescribed value of time $t=t_{0}$, which is necessary for variable SRC, the only quantity needed is the basis coefficients sequence $c_{d}[n]$, assuming that the interpolation kernel $h(t)$ is a known function. From the sampled sequence $x_{d}[n]$ from (4), it can be seen that $c_{d}[n]$ can be obtained as follows.

$$
c_{d}[n]=g_{d}[n] * x_{d}[n]
$$

where $g_{d}[n]$ is the convolutional inverse of $h_{d}[n]$ [7]. In the $z$-domain, (5) becomes,

$$
C_{d}(z)=G_{d}(z) X_{d}(z), \text { where } G_{d}(z)=\frac{1}{H_{d}(z)}
$$

Here, $C_{d}(z), X_{d}(z), G_{d}(z)$, and $H_{d}(z)$ denote the $z$ transforms of $c_{d}[n], x_{d}[n], g_{d}[n]$, and $h_{d}[n]$, respectively. Thus, $c_{d}[n]$ can be obtained using the system shown in Fig. 2 [8].

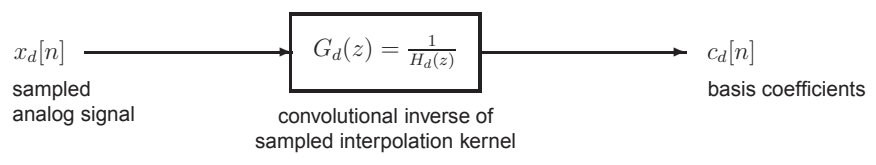

Fig. 2. Discrete-time system used to obtain the interpolation basis coefficients sequence $c_{d}[n]$ from the sampled continuous-time signal $x\left(n T_{s}\right)=x_{d}[n]$.

For the special case in which $x_{d}[n]=c_{d}[n]$, we say that $h(t)$ is interpolating [8]. Otherwise, it is non-interpolating. It can be easily shown that $h(t)$ is interpolating iff we have,

$$
h(n)=\delta[n] \quad \forall n \in \mathbb{Z} \Longleftrightarrow h_{d}[n]=\delta[n]
$$

where $\delta[n]$ is the Kronecker delta function [7].

Combining (5) with (1), it follows that $x(t)$ can be expanded in terms of an effective interpolating kernel $h_{\mathrm{eff}}(t)$ as follows.

$$
x(t)=\sum_{k=-\infty}^{\infty} x_{d}[k] h_{\mathrm{eff}}\left(\frac{t}{T}-k\right)
$$

Here, $h_{\text {eff }}(t)$ is defined as,

$$
h_{\mathrm{eff}}(t) \triangleq \sum_{\ell=-\infty}^{\infty} g_{d}[\ell] h(t-\ell)
$$

From (9), we see that $h_{\mathrm{eff}}(t)$ satisfies (7) and is interpolating. If $H(j 2 \pi F)$ and $H_{\text {eff }}(j 2 \pi F)$ denote the Fourier transforms of $h(t)$ and $h_{\mathrm{eff}}(t)$, respectively, then from (9) and (6), we have,

$$
H_{\text {eff }}(j 2 \pi F)=G_{d}\left(e^{j 2 \pi F}\right) H(j 2 \pi F)=\frac{H(j 2 \pi F)}{H_{d}\left(e^{j 2 \pi F}\right)}
$$

Plots of the impulse and magnitude responses of several effective interpolating kernels are shown in Fig. $3^{1}$ (a) and (b), respectively. All kernels here were interpolating, except for the cubic $B$-spline, for which $H_{d}(z)=\frac{1}{6} z+\frac{2}{3}+\frac{1}{6} z^{-1}$ [9]. As can be seen, higher order methods yielded behavior closer to the bandlimited sinc interpolant of (3) than lower order ones. Specifically, the cubic $B$-spline yielded the closest fit to the sinc interpolant of all of the kernels considered.

\section{The VARIABle SRC Problem}

From (2), the quadrature analog input $x(t)$ to the system of Fig. 1 is assumed to be bandlimited and of the form,

$$
x(t)=\sum_{m=-\infty}^{\infty} x_{d}[m] \operatorname{sinc}\left(\frac{t}{T_{\mathrm{in}}}-m\right)
$$

where $T_{\text {in }}$ denotes the quadrature input sampling interval and $x_{d}[m] \triangleq x\left(m T_{\text {in }}\right)$ denotes the discrete-time sampled signal obtained after baseband down-conversion of the ADC output.

To alter the sample interval to say $T_{\text {out }}$, we need to generate the sequence $y_{d}[n] \triangleq x\left((n+\epsilon) T_{\text {out }}\right)$, where $\epsilon$ is a fractional offset satisfying $0 \leq \epsilon<1$. From (11), $y_{d}[n]$ is calculated as,

$$
y_{d}[n]=\sum_{m=-\infty}^{\infty} x_{d}[m] \operatorname{sinc}(\rho(n+\epsilon)-m)
$$

\footnotetext{
${ }^{1}$ Every interpolating kernel $h_{\mathrm{eff}}(t)$ considered here is real and even meaning that $H_{\text {eff }}(j 2 \pi F)$ is also real and even [7]. As such, all impulse and magnitude responses are shown for $t \geq 0$ and $F \geq 0$, respectively.
} 


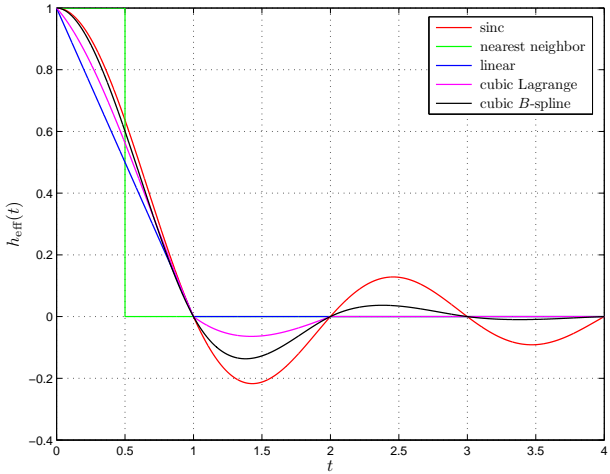

(a)

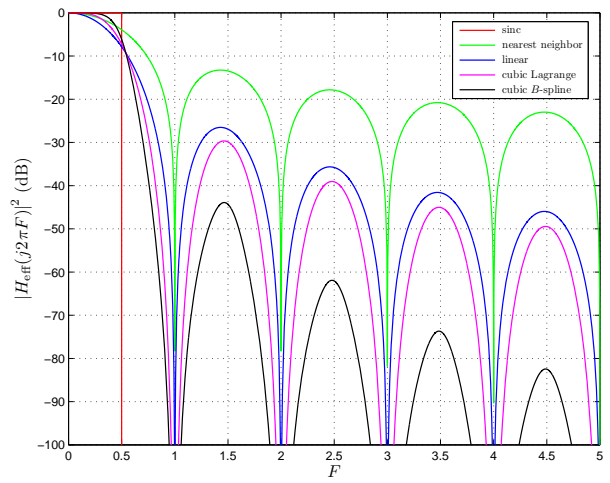

(b)

Fig. 3. Effective interpolating kernel responses: (a) time domain impulse responses and (b) frequency domain magnitude responses.

Here, $\rho$ is defined as $\rho \triangleq \frac{T_{\text {out }}}{T_{\text {in }}}$. If $F_{\text {in }} \triangleq \frac{1}{T_{\text {in }}}$ and $F_{\text {out }} \triangleq \frac{1}{T_{\text {out }}}$ denote, respectively, the input and output sample rates, then $\rho=\frac{F_{\text {in }}}{F_{\text {out }}}$. Hence, $\rho$ is called the input rate conversion ratio. Similarly, $\mathcal{R} \triangleq \frac{1}{\rho}=\frac{F_{\text {out }}}{F_{\text {in }}}$ is the output rate conversion ratio.

To calculate even a single sample of $y_{d}[n]$, the sum in (12) must be computed over the entire set of input samples $\left\{x_{d}[m]\right\}$ in general. For variable SRC in which $\rho$ is adjustable, the sinc terms in (12) must also be changed accordingly. As this cannot be done practically, one alternative could be to truncate most of the terms in (12). However, as the sinc function decays inverse linearly (i.e., $\operatorname{sinc}(x)$ decays as $\left.\frac{1}{x}\right)$, a large number of terms must be kept to minimize truncation error effects [2].

Instead, what is typically done to achieve variable SRC is to model the continuous-time input as coming from a different kernel than the sinc function [3], [4], [5]. Specifically, the input analog signal $v(t)$ is modeled using (1) as follows.

$$
v(t)=\sum_{m=-\infty}^{\infty} c_{d}[m] h\left(\frac{t}{T_{\mathrm{in}}}-m\right)
$$

To alter the sampling interval to $T_{\text {out }}$, we compute $y_{d}[n] \triangleq$ $v\left((n+\epsilon) T_{\text {out }}\right)$ as before. From (13), we get the following.

$$
y_{d}[n]=\sum_{m=-\infty}^{\infty} c_{d}[m] h(\rho(n+\epsilon)-m)
$$

Through careful choice of the kernel $h(t)$, we can achieve variable SRC in (14) efficiently and with little loss compared to the underlying sinc model from (12). For example, for piecewise polynomial kernels, such as the nearest neighbor, linear, cubic Lagrange, and cubic $B$-spline interpolants, $y_{d}[n]$ can be computed efficiently using the Farrow structure [10], [2].

In terms of the effective interpolating kernel $h_{\text {eff }}(t)$, the variable SRC output $y_{d}[n]$ is given as follows from (8).

$$
y_{d}[n]=\sum_{m=-\infty}^{\infty} x_{d}[m] h_{\mathrm{eff}}(\rho(n+\epsilon)-m)
$$

\section{VARiable SRC OUtPut Spectral Properties}

It is insightful to consider frequency domain representations of the variable SRC output $y_{d}[n]$ from (14) and (15). Focusing on (14), if $Y_{d}\left(e^{j 2 \pi f}\right)$ denotes the discrete-time Fourier transform of $y_{d}[n]$, then we have the following [7].

$$
\begin{aligned}
& Y_{d}\left(e^{j 2 \pi f}\right) \triangleq \sum_{n=-\infty}^{\infty} y_{d}[n] e^{-j 2 \pi f n} \\
& =\sum_{m=-\infty}^{\infty} c_{d}[m]\left(\sum_{n=-\infty}^{\infty} h(\rho(n+\epsilon)-m) e^{-j 2 \pi f n}\right)
\end{aligned}
$$

In terms of its inverse Fourier transform [7], $h(t)$ is given by,

$$
h(t)=\int_{-\infty}^{\infty} H(j 2 \pi F) e^{j 2 \pi F t} d F
$$

Using (17) in (16) yields the following after some work.

$$
\begin{aligned}
Y_{d}\left(e^{j 2 \pi f}\right)= & \sum_{m=-\infty}^{\infty} c_{d}[m] \int_{-\infty}^{\infty} H(j 2 \pi F) e^{j 2 \pi F(\rho \epsilon-m)} \\
& \times\left(\sum_{n=-\infty}^{\infty} e^{-j 2 \pi n\left(F-\frac{f}{\rho}\right) \rho}\right) d F
\end{aligned}
$$

Recall the Dirac impulse train [7] Fourier series expansion is,

$$
\sum_{n=-\infty}^{\infty} e^{-j 2 \pi n \widehat{F} \widehat{T}}=\frac{1}{\widehat{T}} \sum_{k=-\infty}^{\infty} \delta\left(\widehat{F}+\frac{k}{\widehat{T}}\right) \quad \forall \widehat{F}, \widehat{T}
$$

Here, $\delta(t)$ is the Dirac delta function [7]. Using (19) in (18) with $\widehat{F}=F-\frac{f}{\rho}$ and $\widehat{T}=\rho$ yields the following after simplification and exploiting the sifting property of $\delta(t)$ [7].

$$
\begin{aligned}
Y_{d}\left(e^{j 2 \pi f}\right)= & \frac{1}{\rho} \sum_{m=-\infty}^{\infty} c_{d}[m] \sum_{k=-\infty}^{\infty} H\left(j 2 \pi\left(\frac{f-k}{\rho}\right)\right) \\
& \times e^{j 2 \pi\left(\frac{f-k}{\rho}\right)(\rho \epsilon-m)} \\
= & \frac{1}{\rho} \underbrace{\sum_{C_{d}\left(e^{j 2 \pi\left(\frac{f-k}{\rho}\right)}\right)}^{\infty} H\left(j 2 \pi\left(\frac{f-k}{\rho}\right)\right) e^{j 2 \pi(f-k) \epsilon}}_{k=-\infty} \\
& \times \sum_{\left.\sum_{d}[m] e^{-j 2 \pi\left(\frac{f-k}{\rho}\right) m}\right)}^{\left(\sum_{i}^{\infty}\right)}
\end{aligned}
$$

Thus, from (20), $Y_{d}\left(e^{j 2 \pi f}\right)$ simplifies to the following form. $Y_{d}\left(e^{j 2 \pi f}\right)=$

$\frac{1}{\rho} \sum_{k=-\infty}^{\infty} H\left(j 2 \pi\left(\frac{f-k}{\rho}\right)\right) C_{d}\left(e^{j 2 \pi\left(\frac{f-k}{\rho}\right)}\right) e^{j 2 \pi(f-k) \epsilon}$ 


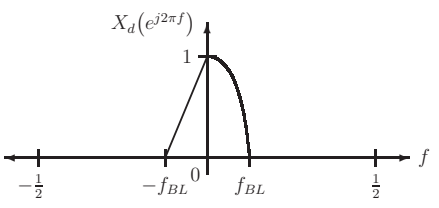

(a)

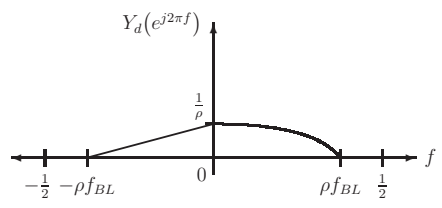

(b)
Fig. 4. Illustration of generalized alias-free decimation via variable SRC: (a) input spectrum before SRC and (b) output spectrum after SRC.

Using (6) and (10) in (21) yields a spectral representation of the variable SRC output from (15) as follows.

$Y_{d}\left(e^{j 2 \pi f}\right)=$

$\frac{1}{\rho} \sum_{k=-\infty}^{\infty} H_{\mathrm{eff}}\left(j 2 \pi\left(\frac{f-k}{\rho}\right)\right) X_{d}\left(e^{j 2 \pi\left(\frac{f-k}{\rho}\right)}\right) e^{j 2 \pi(f-k) \epsilon}$

\section{A. Relation to Decimation}

Suppose $\epsilon=0$ and $h_{\text {eff }}(t)=\operatorname{sinc}(t)$. Furthermore, suppose $x_{d}[n]$ is bandlimited to $f=f_{B L}$, meaning $X_{d}\left(e^{j 2 \pi f}\right)=0$ for $|f| \geq f_{B L}$ for some $f_{B L}$ with $0 \leq f_{B L}<\frac{1}{2}$ and $f \in\left[-\frac{1}{2}, \frac{1}{2}\right)$. If $f_{B L}<\frac{1}{2 \rho}$, where we assume $\rho \geq 1$ here, then from (22),

$$
Y_{d}\left(e^{j 2 \pi f}\right)=\frac{1}{\rho} X_{d}\left(e^{j\left(\frac{2 \pi f}{\rho}\right)}\right), f \in\left[-\frac{1}{2}, \frac{1}{2}\right)
$$

Thus, $Y_{d}\left(e^{j 2 \pi f}\right)$ is a scaled and zoomed in version of $X_{d}\left(e^{j 2 \pi f}\right)$, where the zoom magnification factor is the input rate conversion ratio $\rho$. Note that (23) is a generalization of alias-free decimation [6] for both rational and irrational conversion ratios. An illustration of this is shown in Fig. 4.

Another relation to decimation arises when we set $\epsilon=0$, take $h_{\mathrm{eff}}(t)=\operatorname{sinc}(t)$, and choose $\rho=M$, where $M$ is any positive integer. Using the division theorem [6] to set $k=$ $M q+r$ in (22) (where $q=\left\lfloor\frac{k}{M}\right\rfloor$ and $r=k \bmod M$ ) yields,

$$
Y_{d}\left(e^{j 2 \pi f}\right)=\frac{1}{M} \sum_{r=0}^{M-1} X_{d}\left(e^{j 2 \pi\left(\frac{f-r}{M}\right)}\right)
$$

Note that (24) is the spectrum obtained by decimating $x_{d}[n]$ by $M[6]$. Hence, variable SRC becomes integer decimation through proper choice of the kernel and conversion parameters.

\section{INTERPolation ERROR ANALYSis FOR VARIABLE SRC}

To gauge the difference between using any two kernels, we focus on the mean-squared interpolation error $\zeta$ defined as,

$$
\zeta \triangleq E_{\epsilon}\left[\sum_{n=-\infty}^{\infty}|\gamma[n]|^{2}\right] \text {, where } \gamma[n] \triangleq y_{d}[n]-\widehat{y}_{d}[n]
$$

Here, $y_{d}[n]$ and $\widehat{y}_{d}[n]$ are SRC outputs as in (15) using the same input $x_{d}[m]$ but different kernels $\left(h_{\mathrm{eff}}(t)\right.$ and $\widehat{h}_{\mathrm{eff}}(t)$, respectively). The expectation in (25) is over the offset $\epsilon$, which is assumed to be uniform [11] over [0,1) (i.e., $\epsilon \sim \mathcal{U}[0,1)$ ).

From (15), the error sequence $\gamma[n]$ can be expressed as,

$$
\gamma[n]=\sum_{m=-\infty}^{\infty} x_{d}[m] d(\rho(n+\epsilon)-m)
$$

Here, $d(t) \triangleq h_{\mathrm{eff}}(t)-\widehat{h}_{\mathrm{eff}}(t)$ is the kernel difference signal. As (26) is of the same form as (15), we can use (22) to say, $\Gamma\left(e^{j 2 \pi f}\right)=$

$\frac{1}{\rho} \sum_{k=-\infty}^{\infty} D\left(j 2 \pi\left(\frac{f-k}{\rho}\right)\right) X_{d}\left(e^{j 2 \pi\left(\frac{f-k}{\rho}\right)}\right) e^{j 2 \pi(f-k) \epsilon}$

Here, $\Gamma\left(e^{j 2 \pi f}\right)$ and $D(j 2 \pi F)$ denote the Fourier transforms of $\gamma[n]$ and $d(t)$, respectively.

Returning to (25), from Parseval's theorem [7], $\zeta$ satisfies,

$$
\zeta=E_{\epsilon}\left[\int_{-\frac{1}{2}}^{\frac{1}{2}}\left|\Gamma\left(e^{j 2 \pi f}\right)\right|^{2} d f\right]
$$

Using (27) in (28), we get, after some algebraic manipulation,

$$
\begin{aligned}
& \zeta=\frac{1}{\rho^{2}} \sum_{k=-\infty}^{\infty} \sum_{\ell=-\infty}^{\infty} \int_{-\frac{1}{2}}^{\frac{1}{2}} D\left(j 2 \pi\left(\frac{f-k}{\rho}\right)\right) X_{d}\left(e^{j 2 \pi\left(\frac{f-k}{\rho}\right)}\right) \\
& \times D^{*}\left(j 2 \pi\left(\frac{f-\ell}{\rho}\right)\right) X_{d}^{*}\left(e^{j 2 \pi\left(\frac{f-\ell}{\rho}\right)}\right)\left\{E_{\epsilon}\left[e^{-j 2 \pi(k-\ell) \epsilon}\right]\right\} d f
\end{aligned}
$$

As $\epsilon \sim \mathcal{U}[0,1)$, it can be easily shown that we have,

$$
E_{\epsilon}\left[e^{-j 2 \pi(k-\ell) \epsilon}\right]=\delta[k-\ell]
$$

Substituting (30) into (29) yields the following simplifications.

$$
\begin{aligned}
\zeta= & \frac{1}{\rho^{2}} \sum_{k=-\infty}^{\infty} \int_{-\frac{1}{2}}^{\frac{1}{2}}\left|D\left(j 2 \pi\left(\frac{f-k}{\rho}\right)\right) X_{d}\left(e^{j 2 \pi\left(\frac{f-k}{\rho}\right)}\right)\right|^{2} d f \\
& =\frac{1}{\rho} \sum_{k=-\infty}^{\infty} \int_{\frac{-\frac{1}{2}-k}{\rho}}^{\frac{\frac{1}{2}-k}{\rho}}|D(j 2 \pi \lambda)|^{2}\left|X_{d}\left(e^{j 2 \pi \lambda}\right)\right|^{2} d \lambda \\
& =\frac{1}{\rho} \sum_{m=-\infty}^{\infty} \int_{\frac{m-\frac{1}{2}}{\rho}}^{\frac{m+\frac{1}{2}}{\rho}}|D(j 2 \pi \lambda)|^{2}\left|X_{d}\left(e^{j 2 \pi \lambda}\right)\right|^{2} d \lambda \\
& =\frac{1}{\rho} \int_{-\infty}^{\infty}|D(j 2 \pi \lambda)|^{2}\left|X_{d}\left(e^{j 2 \pi \lambda}\right)\right|^{2} d \lambda
\end{aligned}
$$

Here, (31) follows from the substitution $\lambda=\frac{f-k}{\rho}$, (32) from the summation index $m=-k$, and (33) from the fact that the integration intervals in (32) are nonoverlapping and span $\mathbb{R}$.

To simplify $\zeta$ further, note that from (33), we have,

$$
\begin{aligned}
\zeta & =\frac{1}{\rho} \sum_{\ell=-\infty}^{\infty} \int_{\ell-\frac{1}{2}}^{\ell+\frac{1}{2}}|D(j 2 \pi \lambda)|^{2}\left|X_{d}\left(e^{j 2 \pi \lambda}\right)\right|^{2} d \lambda \\
& =\frac{1}{\rho} \sum_{\ell=-\infty}^{\infty} \int_{-\frac{1}{2}}^{\frac{1}{2}}|D(j 2 \pi(f+\ell))|^{2}\left|X_{d}\left(e^{j 2 \pi(f+\ell)}\right)\right|^{2} d f
\end{aligned}
$$

Here, (34) follows from partitioning the integration interval of (33), while (35) follows from the substitution $f=\lambda-\ell$.

Continuing further, we have the following simplified formula for the mean-squared interpolation error $\zeta$.

$$
\zeta=\frac{1}{\rho} \int_{-\frac{1}{2}}^{\frac{1}{2}}\left(\sum_{k=-\infty}^{\infty}|D(j 2 \pi(f-k))|^{2}\right)\left|X_{d}\left(e^{j 2 \pi f}\right)\right|^{2} d f
$$

Here, (36) follows from the change of summation index $k=$ $-\ell$ and the fact that $X_{d}\left(e^{j 2 \pi f}\right)$ is periodic with period 1 . 


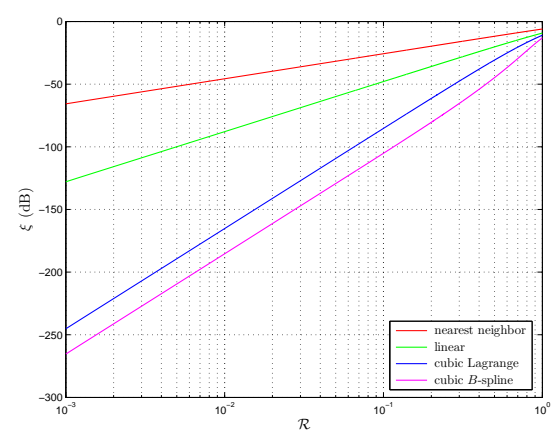

Fig. 5. Normalized mean-squared interpolation error $\xi$ as a function of the output rate conversion factor $\mathcal{R}$ for the input of (39).

\section{Vi. Simulation Results}

For simulation purposes, we will opt to compute the normalized mean-squared interpolation error $\xi$ defined below.

$$
\xi \triangleq \frac{E_{\epsilon}\left[\sum_{n=-\infty}^{\infty}\left|y_{d}[n]-\widehat{y}_{d}[n]\right|^{2}\right]}{E_{\epsilon}\left[\sum_{n=-\infty}^{\infty}\left|y_{d}[n]\right|^{2}\right]}
$$

Here, the kernel $h_{\mathrm{eff}}(t)$ for $y_{d}[n]$ is the sinc function (i.e., $\left.h_{\text {eff }}(t)=\operatorname{sinc}(t)\right)$ and $\widehat{h}_{\text {eff }}(t)$ for $\widehat{y}_{d}[n]$ is some other kernel.

From (36), (37) has the following simplified form.

$$
\xi=\int_{-\frac{1}{2}}^{\frac{1}{2}}\left(\sum_{k=-\infty}^{\infty}|D(j 2 \pi(f-k))|^{2}\right)\left|A\left(e^{j 2 \pi f}\right)\right|^{2} d f
$$

Here, $A\left(e^{j 2 \pi f}\right)$ is a normalized form of $X_{d}\left(e^{j 2 \pi f}\right)$ satisfying $\left|A\left(e^{j 2 \pi f}\right)\right|^{2}=\left|X_{d}\left(e^{j 2 \pi f}\right)\right|^{2} / \int_{-\frac{1}{2}}^{\frac{1}{2}}\left|X_{d}\left(e^{j 2 \pi \lambda}\right)\right|^{2} d \lambda$.

To test the interpolation error in an extremal case, in the spirit of Fig. 4, suppose that $\left|X_{d}\left(e^{j 2 \pi f}\right)\right|^{2}$ is given by,

$$
\left|X_{d}\left(e^{j 2 \pi f}\right)\right|^{2}=\left\{\begin{array}{ll}
\rho, & |f|<\frac{1}{2 \rho} \\
0, & |f| \geq \frac{1}{2 \rho}
\end{array} \quad, f \in\left[-\frac{1}{2}, \frac{1}{2}\right)\right.
$$

Variable SRC in this case corresponds to maximal decimation [6], as there will be no redundancy present in the output signal.

A plot of $\xi$ from (38) for the input of (39) is shown in Fig. 5 as a function of the output conversion factor $\mathcal{R}=\frac{1}{\rho}$ for various kernels. For all methods, as $\mathcal{R}$ increased, $\xi$ increased as well. This is because for larger $\mathcal{R}$, the input samples appear less redundant, and so any given method is more likely to yield output samples that are less consistent with the underlying sinc interpolant. Another observation is that for a fixed $\mathcal{R}$, higher order methods always outperformed lower order ones.

To test the error in a more practical scenario, suppose the input to the system of Fig. 1 is a data stream with a squareroot raised-cosine pulse shape [2]. Then, the spectral density $\left|X_{d}\left(e^{j 2 \pi f}\right)\right|^{2}$ for $f \in\left[-\frac{1}{2}, \frac{1}{2}\right)$ is a raised-cosine pulse with,

$$
\begin{aligned}
& \left|X_{d}\left(e^{j 2 \pi f}\right)\right|^{2}= \\
& \begin{cases}K \rho, & |f|<\frac{1-\alpha}{2 K \rho} \\
K \rho \cos ^{2}\left(\frac{\pi K \rho}{2 \alpha}\left[|f|-\left(\frac{1-\alpha}{2 K \rho}\right)\right]\right), & \frac{1-\alpha}{2 K \rho} \leq|f|<\frac{1+\alpha}{2 K \rho} \\
0, & |f| \geq \frac{1+\alpha}{2 K \rho}\end{cases}
\end{aligned}
$$

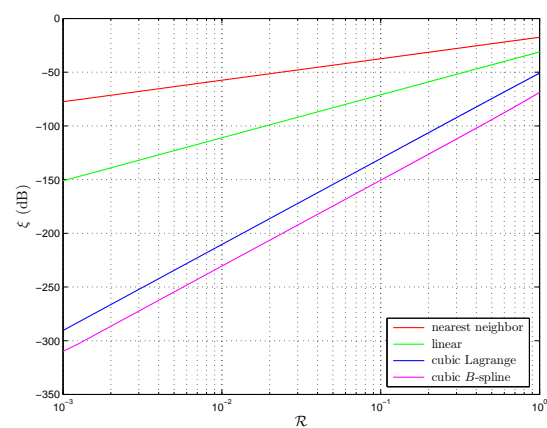

Fig. 6. Normalized mean-squared interpolation error $\xi$ as a function of the output rate conversion factor $\mathcal{R}$ for the input of (40) with $K=4$ and $\alpha=0.35$.

Here, $K$ is the redundancy factor for the tracking loops of Fig. 1 , while $\alpha$ is the roll-off factor for the raised-cosine pulse [11].

As a practical case, suppose $K=4$ and $\alpha=0.35$ [2]. Then, a plot of $\xi$ versus $\mathcal{R}$ for the input of (40) is shown in Fig. 6 for various kernels. As with Fig. 5, when $\mathcal{R}$ increased, $\xi$ increased as well. Also, higher order methods outperformed lower order ones for a fixed $\mathcal{R}$. Comparing Fig. 6 with Fig. 5, the error was lower for the input of (40) than for that of (39). This is because SRC for (40) corresponds to non-maximal decimation due to the extra redundancy required by the tracking loops.

\section{CONCLUDING REMARKS}

In this paper, we derived frequency domain expressions for the variable SRC output and the mean-squared interpolation error. Simulations provided revealed the degradation effects of using a practical interpolation kernel as opposed to the underlying sinc function. Extensions of this analysis to more general interpolation models are the subjects of future research.

\section{REFERENCES}

[1] J. Hamkins and M. K. Simon, Eds., Autonomous Software-Defined Radio Receivers for Deep Space Applications. Hoboken, NJ: John Wiley \& Sons, Inc., 2006.

[2] A. Tkacenko, "Variable sample rate conversion techniques for the Advanced Receiver," Interplanetary Network (IPN) Progress Report, vol. 42-168, pp. 1-33, Feb. 15, 2007.

[3] T. A. Ramstad, "Digital methods for conversion between arbitrary sampling frequencies," IEEE Trans. Acoust., Speech, Signal Process., vol. ASSP-32, no. 3, pp. 577-591, Jun. 1984.

[4] F. M. Gardner, "Interpolation in digital modems - Part I: Fundamentals," IEEE Trans. Commun., vol. 41, no. 3, pp. 501-507, Mar. 1993.

[5] L. Erup, F. M. Gardner, and R. A. Harris, "Interpolation in digital modems - Part II: Implementation and performance," IEEE Trans. Commun., vol. 41, no. 6, pp. 998-1008, Jun. 1993.

[6] P. P. Vaidyanathan, Multirate Systems and Filter Banks. Englewood Cliffs, NJ: Prentice Hall PTR, 1993.

[7] A. V. Oppenheim, R. W. Schafer, and J. R. Buck, Discrete-Time Signal Processing, 2nd ed. Upper Saddle River, NJ: Prentice-Hall, Inc., 1999.

[8] P. Thévenaz, T. Blu, and M. Unser, "Interpolation revisited," IEEE Trans. Med. Imag., vol. 19, no. 7, pp. 739-758, Jul. 2000.

[9] M. Unser, A. Aldroubi, and M. Eden, "Fast $B$-spline transforms for continuous image representation and interpolation," IEEE Trans. Pattern Anal. Mach. Intell., vol. 13, no. 3, pp. 277-285, Mar. 1991.

[10] C. W. Farrow, "A continuously variable digital delay element," in Proc. IEEE International Symposium on Circuits and Systems (ISCAS 1988), Espoo, Finland, Jun. 6-9, 1988, pp. 2641-2645.

[11] M. K. Simon, S. M. Hinedi, and W. C. Lindsey, Digital Communications Techniques: Signal Design and Detection. Upper Saddle River, NJ: Prentice Hall PTR, 1994. 